\title{
ASPP1 and ASPP2 are new transcriptional targets of E2F
}

\author{
V Fogal ${ }^{1}$, NN Kartasheva ${ }^{2}$, G Trigiante ${ }^{1}$, S Llanos ${ }^{1}$, D Yap ${ }^{1}$, \\ $\mathrm{KH}$ Vousden $^{2}$ and $\mathrm{X} \mathrm{Lu} \mathrm{u}^{\star, 1}$ \\ ${ }^{1}$ Ludwig Institute for Cancer Research, University College London, 91 Riding \\ House Street, London W1W 7BS, UK \\ ${ }^{2}$ Beatson Institute for Cancer Research, Garscube Estate, Switchback Road, \\ Bearsden, Glasgow G61 1BD, UK \\ * Corresponding author: X Lu, Ludwig Institute for Cancer Research, University \\ College London, 91 Riding House Street, London W1W 7BS, UK. \\ Tel: + 442078784112; Fax: + 442077248586; E-mail: x.lu@ludwig.ucl.ac.uk
}

Received 25.8.04; revised 16.11.04; accepted 16.11.04; published online 25.02.05 Edited by G Melino

\begin{abstract}
The E2F family of transcription factors regulates the expression of a number of genes whose products are involved in cell cycle control, DNA replication and apoptosis. We show here that E2F-1 binds in vivo the promoters of ASPP1 and ASPP2 genes, two activators of p53-mediated apoptosis, E2F-1, E2F-2 and E2F-3 all activate the isolated ASPP1 and ASPP2 promoters. Overexpression or deregulation of E2F-1 increased the expression levels of ASPP1 and ASPP2 mRNA and proteins. The identification of ASPP1 and ASPP2 genes as transcriptional targets of E2F provides another mechanism by which E2F cooperates with p53 to induce apoptosis.

Cell Death and Differentiation (2005) 12, 369-376.

doi:10.1038/s..cdd.4401562

Published online 25 February 2005
\end{abstract}

Keywords: E2F; ASPP; promoter; transcriptional activation; apoptosis; cancer; p53

Abbreviations: $\mathrm{Rb}$, retinoblastoma gene/protein; ASPP, apoptosis-stimulating proteins of p53; ES, E2F DNA binding site; ChIP, chromatin immunoprecipitaion; wt, wild-type; luc, luciferase reporter gene; $\beta$-gal, $\beta$-galactosidase; FCS, foetal calf serum; $\mathrm{RPE}$, retinal pigment epithelial cells

\section{Introduction}

Mutated in more than half of human cancers, ${ }^{1}$ p53 remains one of the most important tumour suppressors identified so far. In response to multiple environmental stresses, p53 is rapidly activated and it functions in inhibiting cell growth either by the induction of cell cycle arrest or apoptosis. However, the tumour suppressor function of p53 is perhaps best linked to its ability to induce apoptosis. ${ }^{2-4}$ Interestingly, the activation of p53 in normal cells usually results in cell cycle arrest, whereas in transformed cells it often induces cell death. ${ }^{5}$ This provides an ideal base to specifically kill wild-type (wt) p53 expressing tumour cells. However, the precise determinants for the sensitivity of tumour cells to p53-induced apoptosis are not yet fully understood.

Tumorigenesis in many cell types involves the inactivation of the retinoblastoma $(\mathrm{Rb})$ pathway. One of the primary consequences of $\mathrm{Rb}$ inactivation is the deregulated activity of E2F. The E2F family of transcription factors plays a central role in regulating cellular proliferation by promoting the expression of genes required for cell cycle progression. Importantly, in addition to inducing proliferation, deregulated E2F activity can also trigger apoptosis via both p53dependent and -independent pathways. ${ }^{6-8}$ Therefore, it is possible that part of the apoptotic sensitivity of tumour cells is due to the ability of E2F-1 to stimulate the apoptotic function of p53. One molecular mechanism that has been associated with E2F-induced p53 dependent apoptosis involves the induction of the ARF tumour suppressor gene by E2F. ${ }^{9}$ ARF interacts with Mdm2 and inhibits its ability to target p53 for ubiquitination and subsequent degradation. ${ }^{10}$ However, results from studies of mutant mouse models indicate that E2F can induce p53-dependent apoptosis in the absence of ARF. ${ }^{11,12}$ Although this does not rule out a role for ARF in the E2F-mediated activation of p53, it suggests the existence of ARF-independent pathways in mediating apoptosis induced by E2F and $\mathrm{p} 53$.

The discovery of the apoptosis-stimulating proteins of $p 53$ (ASPP) family of proteins revealed a key mechanism through which the apoptotic function of $p 53$ is regulated. ${ }^{13,14}$ The ASPP family consists of three members, ASPP1, ASPP2 and iASPP, and they all bind $p 53$ via their $\mathrm{C}$-terminus. ASPP1 and ASPP2 stimulate, whereas IASPP inhibits, the apoptotic function of p53 and its family members, p63 and p73, ${ }^{15}$ both in vitro and in vivo. The importance of the ASPP/p53 regulation is reflected by the observation that Caenorhabditis elegans iASPP and human iASPP are interchangeable. ${ }^{14}$ The human ASPP family members also regulate the apoptotic function of $C$. elegans p53 in a similar way as that of human p53. ${ }^{14}$ We thus investigated whether E2F can cooperate with p53 to induce apoptosis by regulating the expression of the ASPP family of proteins, since an increased expression of ASPP2 in response to E2F-1 activation was previously reported in a gene array study. ${ }^{16}$

\section{Results}

\section{ASPP1 and ASPP2 promoters contain putative E2F binding sites}

To understand how the expression of the ASPP1 and ASPP2 genes are regulated in vivo, promoters of ASPP1 (-736 to $+96)$ and ASPP2 $(-323$ to +217) were isolated from genomic DNA and scanned for the presence of theoretical E2F binding sites using the method described by Kel et al. ${ }^{17}$ (and Materials and Methods). By means of this software, we were able to identify potential E2F binding sites both on the promoters of ASPP1 (four sites) and ASPP2 (two sites) 
(Figure 1). These findings suggested that ASPP1 and ASPP2 might be potential transcriptional targets of E2F.

\section{E2F-1 binds ASPP1 and ASPP2 promoters in vivo}

To determine whether E2F-1 binds to the promoter regions of ASPP1 and ASPP2 genes, we performed a chromatin immunoprecipitation assay (ChIP) in Saos-2 cells inducible for the expression of wt E2F-1. ${ }^{18}$ As shown in Figure $2 a$, over $80 \%$ of the cells expressed inducible E2F-1 at high levels in comparison to the uninduced cells. Anti-E2F-1 antibody C-20 was used to immunoprecipitate formaldehyde crosslinked E2F-1-DNA complexes derived from Saos-2 cells expressing endogenous or overexpressed E2F-1. The presence of ASPP1 and ASPP2 promoter sequences in the immunoprecipitates was verified by PCR using primers surrounding the identified putative E2F binding sites within the ASPP1 and ASPP2 promoters. As shown in Figure 2b, ASPP1 and ASPP2 promoter sequences were specifically detected in the immunoprecipitates derived from E2F-1 antibody but not from the negative control rabbit immunoglobulin, suggesting that endogenous E2F-1 binds ASPP1 and ASPP2 promoters in vivo. Consistent with this, increased amounts of ASPP1 and ASPP2 promoter sequences were also detected in cells expressing inducible E2F-1 (Figure 2b). These results provided a strong positive confirmation for the functional significance of the predicted E2F binding sites within the ASPP1 and ASPP2 promoters and suggested that ASPP1 and ASPP2 may be novel targets of E2F-1 in vivo.

\section{E2F-1 transactivates the promoters of ASPP1 and ASPP2}

To investigate whether E2F-1 could activate the isolated ASPP1 and ASPP2 promoters, the ASPP1 and ASPP2 promoter fragments containing the putative E2F binding sites were cloned into a luciferase reporter plasmid. The responsiveness of these promoters to E2F-1 was then tested in Saos-2 cells. Increasing amounts of E2F-1 stimulated the activity of both ASPP1 and ASPP2 promoters (Figure 3a). At the highest concentration, the extent of activation on the ASPP1 promoter by E2F-1 was almost comparable to a wellknown artificial promoter containing three E2F binding sites $(3 \times$ wt-luc (luciferase reporter gene)). Interestingly, the responsiveness of the ASPP2 promoter to E2F-1 was much higher than that of $3 \times$ wt-luc (Figure $3 a$ ). The transactivation function of $\mathrm{E} 2 \mathrm{~F}-1$ was required to stimulate the transcriptional activity of ASPP1 and ASPP2 promoters since a transcriptionally defective E2F-1 mutant, E2F-1(1-400), did not effect the promoter activity of ASPP1 and ASPP2 (Figure 3b).

We focused our studies on the identification of the critical E2F binding site within the ASPP1 promoter since the

\begin{abstract}
a
$-736$

ES 1

GGGATGCTGGCGCAGGTGGGCACAGGTGGCACAGGTGGGCGCGGGGTTCTAGGCGGGAGATCGCTCGGGCGAAGGCCAAGGCTCGGGA GCTGGTGCGGGGGGCGCGGCCCCGAGAGCCGCGGAAGACGCCGGTGGGGGAGAAGCAGGTGCCAGCCAAGGGCGTCGGGCCGGGGCTGGTTCCG AGCGCGCGGCCTCGGGTCACATGCTCGCTCCGGGGGCCCCCCGCGCCCCTCCCACTGGCCTCTGGGCCGCAGCCCCACGGCGCGCCGGCAGCCGGG GGTCAGGCCGCGGTCAGAGTGCGGGCGCGCGCCGTCGCCGCAGGGACGCTCCCGGCCCACAGTCGGCCCCCGGGAGCCCGGGTTCGAGTCCCGGCC CTGCCGTGATCCGCTGCGGGACCCGGGCCGGCCCTTCCCTGTCGGGCCTCAGTCCTCCCACCGTCCCTGGTCTTCCCTAGTCGGGGCTCACGCAAGGC CGGGGGAGGCCACCGGTGCCCACTGCGCGTCCGCCCCTCCCCCGCCGCCGGGAGCGCGGACGCGCACGCACCTGGCCGCCGCTGAGAGCGAGGGTA ES 2

GCGGGCGGAGCGCGGAAGCCCCGCCCCTCTCCCCGCCCCCGCTCCCACCGGGCCAATCAGAGGGGGCTACGGGACCCGTGCGG $\underline{\text { GGCG }}$ ES 3 TCGCGCTGGGGGCGGGGCCTGCGGGGGCTGAAGAGCGAGCCGGGCGGACGGGCCGCAGGCTGTCGGGCTGGGGCTGAGGCTGAGGCTGA ES 4 GGTTGAGGCGGCGGCGGCGGCGGCCGGGTGCCCGGGACAGCGACGCAGCGCGCCGGCGGCCGCGACAGGGCCAGCGAGAGCCCCGCAG
\end{abstract}

\title{
b
}

$-323$

GGAGCCGCCGAGGAGGTGACGGCGGCAGCCCTGGGGCGGCTCGGTGCCATTCACTACCATACCTGTTTTACCTGAGGCGCGGCCTTCCAATTGCCCC GGCTCCGCTAGGAGGCGCGCGCTCCAGCCGCTCCGCCGCGCGGCCCCGACCGCACTTCAGTCCGGGGCGAAGAAAGAAAAGGCGGCCGGCTCCCG ES 1 CCGCTCCCCGTCTACCCAGCCGGGCCCGCGCAGGCGCGCGCAGCTCCCGTTCCCGCGGCCGCCCCTCCCCCAGGCCGCCGGGCGCGCCGCG ES 2 $\longrightarrow$

GGCGGGGTCGGCGCGGGGGGCGGAGCCGGCACGGGCTCGGCTGGGGCCCGACCCGGGATTAGTTGGTTTCGGAGCGGAGGAGGGAGCCC CGACCGTCACGAGCGTCGAAGAGACAAAGCCGCGTCAGGGGGCCCGGCCGGGGCGGGGGAGCCCGGGGCTTGTTGGTGCCCCAGCCCGCGCGGAG GGCCCTTCGGACCCGCGCGCCGCCACTGCCGCCGCCGCCGCCTCGCAACAGGTCCGGGCGGCCTCGCTCTC

Figure 1 Sequence of the human ASPP1 and ASPP2 promoters. The nucleotide sequence of the ASPP1 promoter (a) and ASPP2 promoter (b) are shown. The transcription starts $(+1)$, indicated by arrows, refer to the longest $5^{\prime}$ published ASPP1 and ASPP2 cDNA sequences (GeneBank gi 21848243 for ASPP1 and gi 30774493 for ASPP2). Putative DNA binding consensus sites for E2F predicted by the SiteScan program are shown in bold and underlined 
a

E2F-1
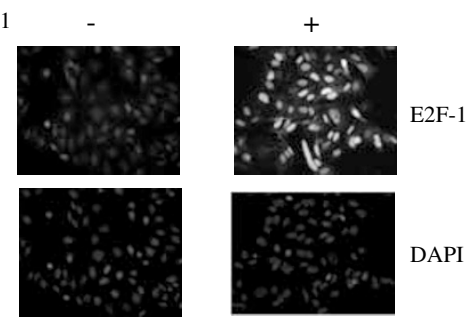

E2F-1

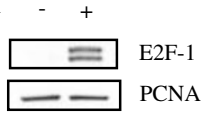

b

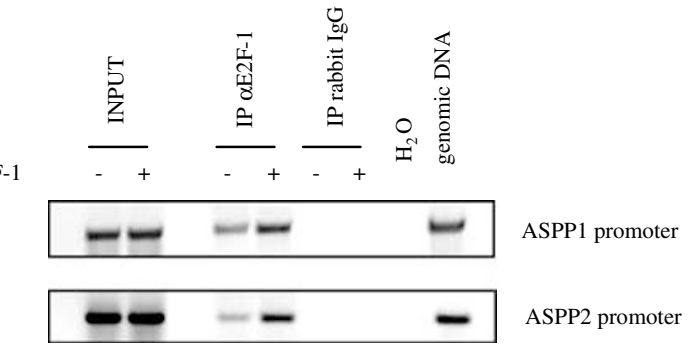

Figure 2 E2F-1 binds to the ASPP1 and ASPP2 promoters in vivo. (a) The levels of induced E2F-1 in Saos-2 cells were detected by immunostaining and immunoblotting using the polyclonal anti E2F-1 antibody C-20. Nuclei were stained by DAPI. PCNA was used as loading control. (b) ChIP assay detected the presence of ASPP1 and ASPP2 promoter sequences only in the formaldehyde crosslinked Saos-2 immunoprecipitates pulled down by the anti-E2F-1 antibody but not by the control rabbit immonoglobulin ( $\mathrm{lgG}$ ) as indicated. DNA derived from aliquots of cell lysates were labelled as INPUT. $\mathrm{H}_{2} \mathrm{O}$ and Saos-2 genomic DNA served as negative and positive controls for the PCR, respectively

accompanying paper by Chen et al. performed a detailed analysis of the ASPP2 promoter. Various truncation mutants of the ASPP1 promoter were generated and their transcriptional activity was measured (Figure 3c). The smallest fragment that retained most of the basal transcriptional activity of ASPP1 promoter was ASPP1 (-146/+96)-luc. There was a significant reduction in the transcriptional activity with ASPP1 $(-113 /+96)-$ luc. Thus, the most critical region for the basal transcriptional activity of the ASPP1 promoter locates within the $(-146 /-113)$ region. Consistent with this, the ASPP1 $(-182 /+19)$-luc had transcriptional activity similar to ASPP1 $(-146 /+96)$-luc, whereas ASPP1 $(-1 /+96)$-luc was as active as ASPP1 $(-113 /+96)$-luc (Figure $3 c$ ). Although two of the putative E2F binding sites (E2F sites 3 and 4) were retained in ASPP1 $(-146 /+96)$-luc, ASPP1 $(-113 /+96)$-luc and ASPP1 $(-1 /+96)$-luc, only ASPP1 $(-146 /+96)$-luc maintained the full basal transcriptional activity. Hence, it is unlikely that E2F is the transcription factor responsible for the basal transcriptional activity of the ASPP1 promoter. Interestingly, however, while the expression of E2F-1 had a detectable but modest effect on the transcriptional activity of ASPP1 $(-736 /+96)$-luc, ASPP1 $(-565 /+96)$-luc and ASPP1 $(-146 /+96)$-luc, the expression of E2F-1 stimulated ASPP1 $(-1 /+96)$-luc by over 20 -fold. These results suggest that E2F-1 may use the binding site located within ASPP1 $(-1 /+96)$-luc (E2F site 4$)$ to stimulate the activity of the ASPP1 promoter. In agreement with this hypothesis, the expression of E2F-1 failed to have any effect on the transcriptional activity of ASPP1 $(-182 /+19)$-luc, a fragment that does not contain the E2F binding site 4 . These results suggested that E2F-responsive elements are likely to be located within the $(+20 /+96)$ region of the ASPP1 promoter.

\section{ASPP1 and ASPP2 are transcriptional targets of E2F-1, E2F-2 and E2F-3}

While some reports recognise E2F-1 as the only member of the family capable of promoting apoptosis, ${ }^{19-21}$ other studies have shown high levels of cell death in cells expressing active $\mathrm{E} 2 \mathrm{~F}-1, \mathrm{E} 2 \mathrm{~F}-2$ or E2F-3. ${ }^{22}$ It was therefore important to investigate whether ASPP1 and ASPP2 are specific targets of $\mathrm{E} 2 \mathrm{~F}-1$ or their transactivation is a property shared by all three E2F. Expression plasmids of E2F-1, E2F-2 and E2F-3 were first titrated to obtain the expression level that activated the control $3 \times$ wt-luc reporter with similar efficiency. Under these conditions, the expression of E2F-1, E2F-2 and E2F-3 led to comparable increases in ASPP2 promoter activity (Figure 4), suggesting that all three E2F transcriptional activators can efficiently stimulate the activity of the ASPP2 promoter. Similarly, the promoter activity of ASPP1 was also induced by the expression of E2F-1, E2F-2 and E2F-3.

\section{Increased expression of E2F-1 upregulates endogenous ASPP1 and ASPP2 mRNA and protein levels}

To demonstrate that E2F-1 induces the expression of endogenous ASPP1 and ASPP2, total RNA was extracted from Saos-2 and $\mathrm{H} 1299$ cells inducible for the expression of E2F-1. RT-PCR was then performed to detect the expression levels of ASPP1 and ASPP2 mRNA. The induced expression of E2F-1 resulted in a significant increase in ASPP1 and ASPP2 mRNA and protein levels in Saos-2 cells (Figure 5a). Similar results were also obtained in $\mathrm{H} 1299$ cells (Figure $5 b$ ). Furthermore, the transcriptional activity of E2F-1 is required to induce the expression of ASPP1 and ASPP2 since the transcriptional defective E2F-1 mutant, E2F-1 (1-400), failed to induce the expression of ASPP1 and ASPP2 (Figure 5c). These data indicated that ASPP1 and ASPP2 are transcriptional targets of E2F-1 in vivo.

\section{Endogenous E2F can elevate the expression of ASPP1 and ASPP2}

To provide evidence that ASPP1 and ASPP2 are regulated by endogenous E2F, Saos-2 cells were cultured for $48 \mathrm{~h}$ in medium supplemented with 0.1 or $20 \%$ serum to obtain arresting or cycling cells (Figure 6a). The expression level of endogenous E2F-1 was much higher in cycling cells (Figure 6a, top panel, compare lanes 1 and 3) and this correlated with an increase in the expression levels of ASPP1 and ASPP2 mRNA and protein (Figure 6a, wb and RT-PCR panels, compare lanes 1 and 3). Upon the induction of E2F-1 expression, the increase of ASPP1 and ASPP2 mRNA and protein levels was also more prominent in Saos-2 cells 
a

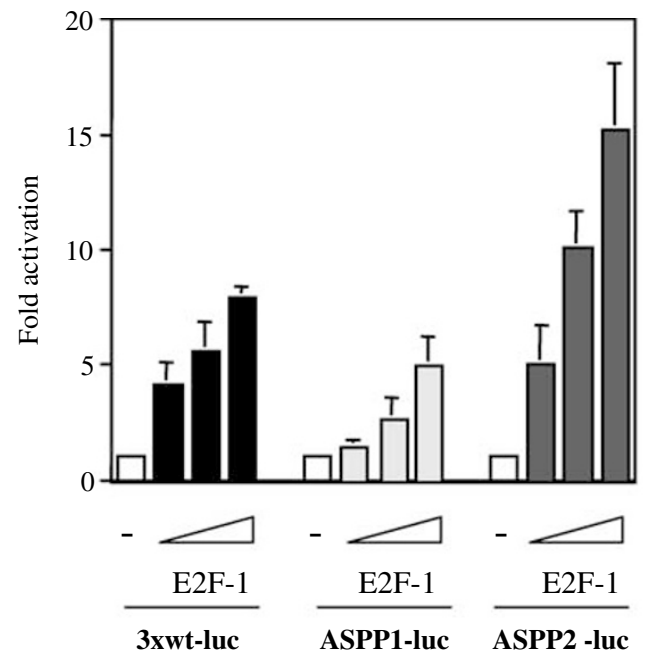

b
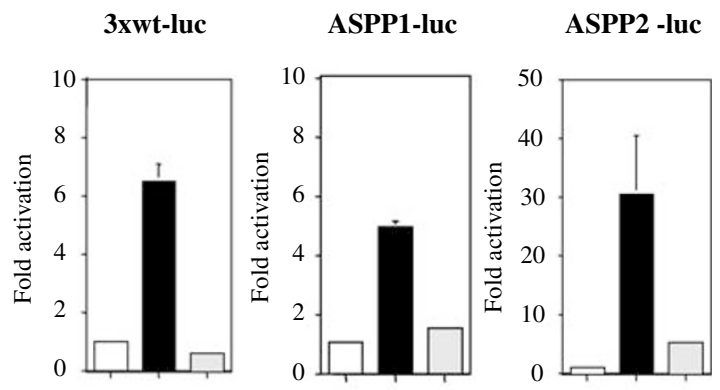

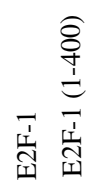

wb 9E10
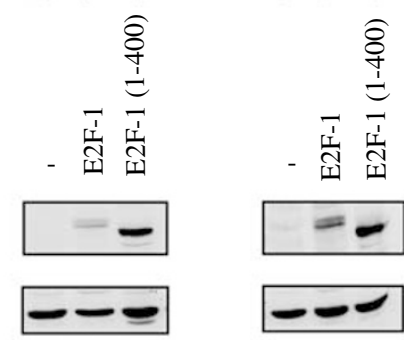

PCNA
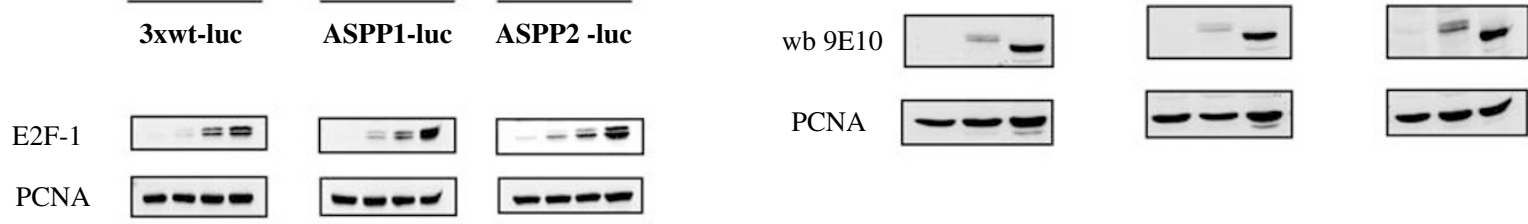

C
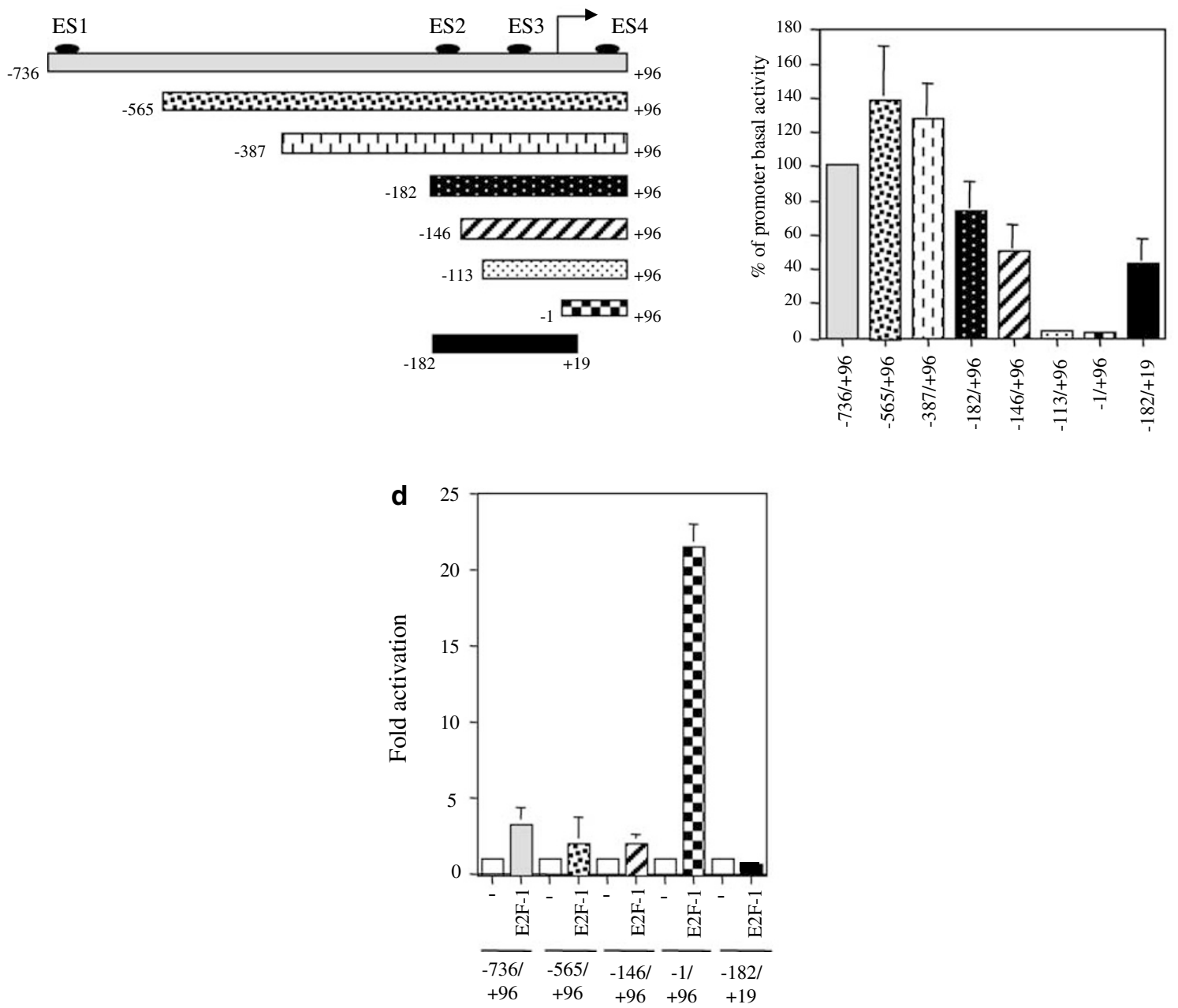


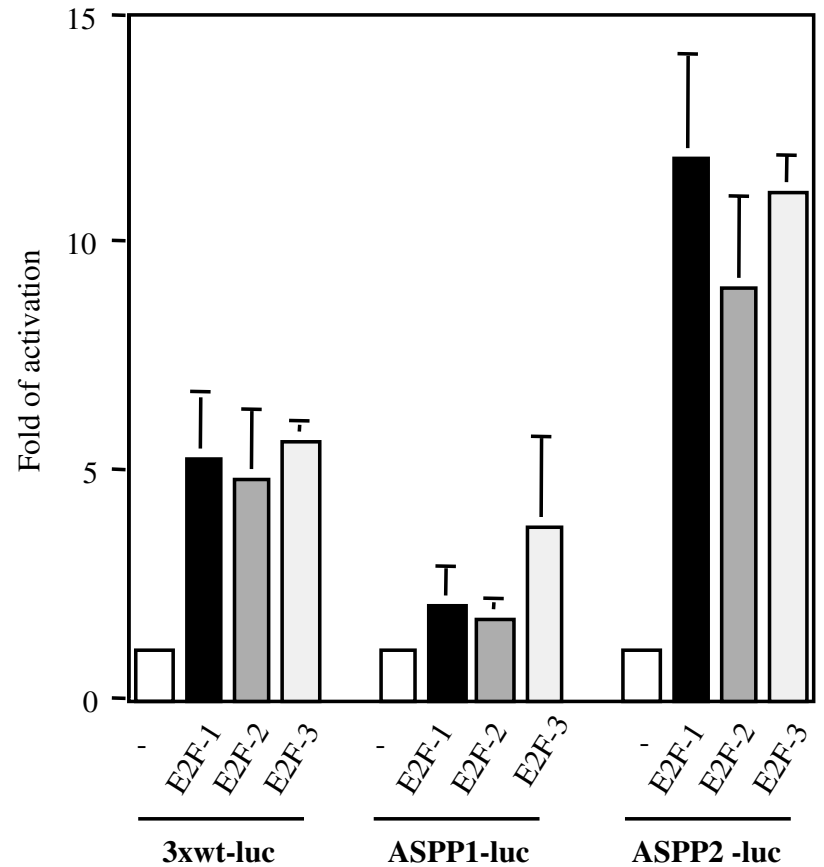

Figure 4 E2F-1, E2F-2 and E2F-3 activate the ASPP promoters. Saos-2 cells were transfected with $\mathrm{pCMV} \beta$-Gal, ASPP1-luc or ASPP2-luc together with E2F$1, \mathrm{E} 2 \mathrm{~F}-2$ or E2F-3 at the concentrations of $0.15,1$ and $0.5 \mu \mathrm{g}$, respectively. The bar graph depicts fold activation in the luciferase assay after normalisation for $\beta$-gal activity, and was derived from three independent experiments

growing in $20 \%$ serum than those in $0.1 \%$ serum (Figure $6 a$, compare lanes 2 and 4 ).

By binding to the $\mathrm{Rb}$ protein, oncogenes like $\mathrm{E} 1 \mathrm{~A}$ dissociate $\mathrm{Rb} / \mathrm{E} 2 \mathrm{~F}$ complexes leading to the deregulation of endogenous E2F activity. The expression levels of endogenous ASPP1 and ASPP2 were measured in human retina pigment epithelial cells (RPE) expressing either a control vector or E1A. An increased expression of a known E2F target gene, $p 73,{ }^{23,24}$ was detected in the E1A expressing RPE cells but not in the control cells. Similarly, the expression of E1A also induced the expression of ASPP1. Interestingly, however, under the same conditions, the presence of E1A had much less effect on the expression levels of ASPP2 (Figure 6b). These results suggest that elevated E2F activity is sufficient to increase the expression of ASPP1 and ASPP2 in vivo.

\section{Discussion}

Existing studies showed that E2F cooperates with p53 to induce apoptosis in vitro and in vivo. There are many pathways through which E2F can increase p53-mediated apoptosis. E2F-1, E2F-2 and E2F-3 can increase the apoptotic function of $\mathrm{p} 53$ through protein-protein interaction. ${ }^{25}$ By transactivating p14Arf (p19Arf in mouse), E2F can also increase the expression levels of p53 since Arf binds Mdm2 and prevents Mdm2-mediated degradation of p53. Additionally, E2F and p53 can also transactivate common target genes like Apaf-1, ${ }^{26,27}$ PUMA and Noxa, ${ }^{28}$ and therefore cooperate in the induction of apoptosis. The identification of ASPP1 and ASPP2 as transcriptional targets of E2F provides another pathway by which E2F increases the apoptotic function of $\mathrm{p53}$, since ASPP1 and ASPP2 are specific activators of p53-mediated apoptosis. ${ }^{13}$ In addition, ASPP1 and ASPP2 are also activators of p63 and p73. ${ }^{15}$ Thus, E2F may use this pathway to increase the apoptotic function of p63 and p73 independently of p53.

Using a recently described computer-assisted tool for the identification of potential E2F binding sites, ${ }^{17}$ we identified putative E2F binding sites within ASPP1 and ASPP2 promoter sequences ( 4 and 2 , respectively). ChIP experiments allowed us to demonstrate that endogenous and exogenous E2F-1 can bind both ASPP1 and ASPP2 promoter sequences in vivo. Consequently, increased expression of E2F-1 also induced the expression of ASPP1 and ASPP2 at both mRNA and protein levels. Based on the truncation studies of the ASPP1 promoter, we hypothesised that the E2F binding site located within the untranscribed region of exon 1 might be the main E2F-responsive site since the most responsive fragment of ASPP1 contains only this site. Further studies are required to test this hypothesis, especially in light of the ASPP2 promoter analysis performed by Chen et al. in the accompanying paper, which indicates a complex regulation of the transcriptional activity of the ASPP2 promoter by E2F.

Nevertheless, the results shown here demonstrate that ASPP1 and ASPP2 are novel transcriptional targets of E2F. Inactivation of $\mathrm{Rb}$ pathways is one of the most common defects found in human cancers. Therefore, most of the tumour cells will have increased E2F activity and consequently an increased expression of ASPP1, ASPP2 or both. In agreement with this, increased expression of ASPP1 was detected in RPE cells expressing the E1A oncogene, which is known to prevent $\mathrm{Rb}$ from inhibiting the transcriptional activity of E2F. Importantly, E1A was previously shown to sensitise mouse primary fibroblast cells to p53-mediated apoptosis. ${ }^{29}$ Perhaps increased expression of ASPP1 or ASPP2 is one of the underlining mechanisms by which E1A enhances the cellular sensitivity to p53-mediated apoptosis. Similar mechanisms may also explain at least in part why most tumour cells are more sensitive to p53-mediated apoptosis than their normal counterparts.

Figure 3 Transcriptional activity of E2F-1 is required to activate ASPP1 and ASPP2 promoters. Saos-2 cells were transfected with the internal control CMV- $\beta$-Gal $(500 \mathrm{ng})$ and $1 \mu \mathrm{g}$ of ASPP1 or ASPP2 promoter reporters. Co-expression of empty vector (-) and increasing amounts of E2F-1 (75, 150 or 300 ng) (a) or E2F-1 (1-400) $(300 \mathrm{ng})(\mathrm{b})$ are indicated. The $3 \times \mathrm{E} 2 \mathrm{~F}$ luciferase reporter $(3 \times$ wt-luc) was used as a positive control. Luciferase values were corrected for transfection efficiency by $\beta$-gal assay. The bar graph shows the fold activation of ASPP1-luc, ASPP2-luc and $3 \times$ wt-luc in the presence or absence of E2F-1 or E2F-1 (1-400). Lower panels of (a) and (b) show the expression levels of 9E10-tagged E2F-1 or E2F-1 (1-400). The PCNA blot served as loading control. (c) (Left) Schematic representation of the ASPP1 promoter and its deletion constructs. The transcription start site is indicated with an arrow. Putative E2F binding sites 1-4 are indicated as ES1, ES2, ES3 and ES4. (Right) Saos-2 cells were transfected with luciferase reporter plasmids containing full-length ASPP1 promoter or its deletion mutants. The effects of E2F-1 expression (patterned bars) on the selected panel of ASPP1 promoter constructs are shown in (d). Bar graphs were derived from at least three independent experiments 
a

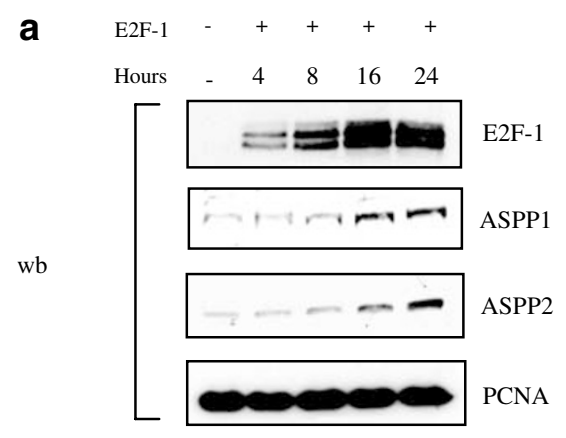

b

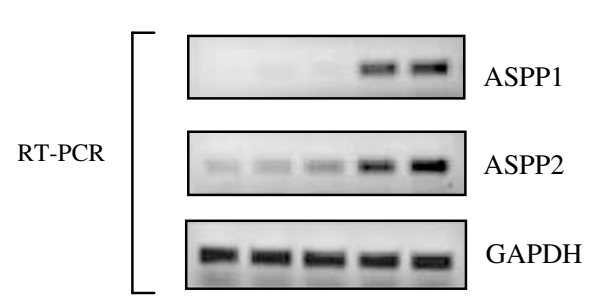

c
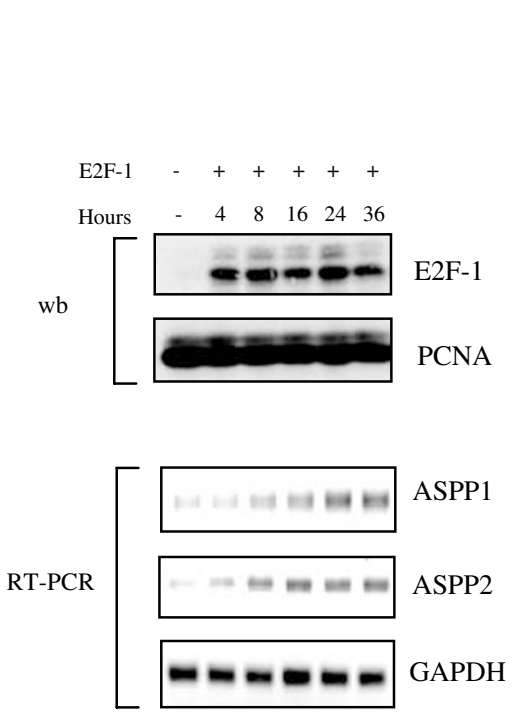

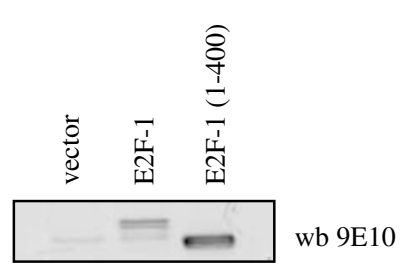

$\longrightarrow$ wb PCNA

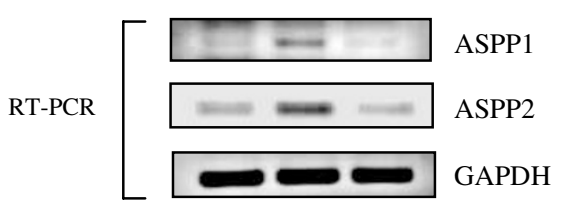

Figure 5 E2F-1 induces the expression of endogenous ASPP1 and ASPP2 mRNA and protein. Saos-2 (a) and H1299 (b) E2F-1 inducible cell lines were treated with doxycycline $(2 \mu \mathrm{g} / \mathrm{ml})$ and ponasterone $\mathrm{A}(10 \mu \mathrm{M})$, respectively, for the indicated hours to induce the expression of E2F-1. Antibodies C-20, mASPP1/54.2 and mASPP2/ 54.10 were used to detect the expression of E2F-1, ASPP1 and ASPP2, respectively (first four panels). PCNA was used as a loading control. RT-PCR was performed using total RNA extracted from cells at the indicated hours after the induction of E2F-1. Primers specific to ASPP1 or ASPP2 were used to amplify ASPP1 or ASPP2 CDNA. The expression of GAPDH gene was used to ensure that equal amounts of total cDNA were used for each time point. Basal levels of ASPP1 and ASPP2 mRNA and proteins were detected in uninduced cells (-). The transcription defective E2F-1 (1-400) failed to induce the expression of ASPP1 and ASPP2 mRNA in Saos-2 cells (c)

\section{Materials and Methods}

\section{Cloning of the ASPP1 and ASPP2 promoters and plasmids}

The ASPP1 and ASPP2 promoters were amplified from human genomic DNA using primers specific for ASPP1 or ASPP2 sequences listened below. The fragments were cloned into pCDNA-3.1-His-V5-TOPO (Invitrogen) and later excised by digestion and subcloned into pGL3Basic (Promega). The resulting constructs comprise the -736 to +96 promoter region of ASPP1 and the -323 to +217 promoter region of ASPP2 (measured relative to the longest $5^{\prime}$ EST currently available, GeneBank gi 21848243 and gi 30774493 for ASPP1 and ASPP2, respectively).

\section{ASPP1: forward 5'-GAACCAGACAGGCTCCAGACTGACCCT-3' reverse 5'-GCTGCGGGGCTCTCGCTGG-3' \\ ASPP2: forward 5'-GCTCGGTGCCATTCACTACCATACC- $3^{\prime}$ reverse $5^{\prime}$-GCTTTGTCTCTTCGACGCTCGTG- $3^{\prime}$}

\section{Identification of putative E2F binding sites within ASPP1 and ASPP2 promoters}

The ASPP1 and ASPP2 promoters were scanned for likely E2F response elements by means of the SiteScan search algorithm (http://compel.bionet.nsc.ru/FunSite/SiteScan.html) as described in Kel et al. ${ }^{17}$ By using this software, we identified two E2F binding sites on the ASPP2 promoter using the weight matrix with score of context option and a $q$ parameter of 0.8 and four E2F binding sites on the ASPP1 promoter using the plain weight matrix method and a $q$ value of 0.81 .

\section{Cell culture, antibodies and plasmids}

Saos-2, H1299 and RPE cells were grown in DMEM supplemented with $10 \%$ foetal calf serum (FCS). Full-length E2F-1 cDNA and E2F-1(1-400) were cloned into the pcDNA-3 expression plasmid (Invitrogen) in frame with the 9E10 epitope tag at the N-terminus. The 9E10 epitope was recognised by the mouse monoclonal antibody $9 \mathrm{E} 10$. The mouse monoclonal antibody $\mathrm{PC}-10$ is specific to the proliferating cell nuclear antigen (PCNA) protein. C-20, an anti-E2F-1 polyclonal antibody used in the immunoprecipitation assays, was purchased from Santa Cruz Biotechnology. Expression plasmids used in this study were driven by the CMV immediate-early promoter.

\section{E2F-1 inducible H1299 and Saos-2 cell lines}

Saos-2 cells expressing inducible E2F-1 under the PTET-On system were described previously. ${ }^{18}$ In H1299 cells, E2F-1 expression was controlled under ponasterone $\mathrm{A}$. This cell line was constructed according to the manufacturer's specifications (Invitrogen, USA).

\section{ChIP assay}

Cells were crosslinked with 1\% formaldehyde (Fisher Scientific) in PBS for $10 \mathrm{~min}$ at room temperature. The crosslinking reaction was stopped by adding glycine to a final concentration of $0.125 \mathrm{M}$. Crosslinked cells were washed twice with cold PBS, trypsinised, washed with PBS and lysed in $500 \mu$ l of RIPA buffer ${ }^{30}$ containing a protease inhibitor cocktail (Complete Mini, Roche), $\mathrm{NaF}(50 \mathrm{mM}), \mathrm{Na}_{3} \mathrm{VO}_{4}(1 \mathrm{mM})$ and $\beta$-glycerophosphate $(10 \mathrm{mM})$. Samples were sonicated with a Heat Systems sonicator (six bursts of $15 \mathrm{~s}$ each) on ice, and centrifuged at $20000 \times g$ for $10 \mathrm{~min}$. Samples $(2-4 \mathrm{mg}$ ) of protein extracts were precleared with $10 \mu$ l of Protein 
G Sepharose beads (preblocked with $1 \mu \mathrm{g} / \mu \mathrm{lBSA}$ and $1 \mu \mathrm{g} / \mu \mathrm{l}$ salmon sperm DNA) for $2 \mathrm{~h}$ at $4^{\circ} \mathrm{C}$ and immunoprecipitated overnight at $4^{\circ} \mathrm{C}$ with $2 \mu \mathrm{g}$ of anti E2F-1 C-20 antibody. Rabbit IgG $(2 \mu \mathrm{g})$ were used as negative control for the immunoprecipitation. Immunoprecipitates were washed

\section{a}

FCS
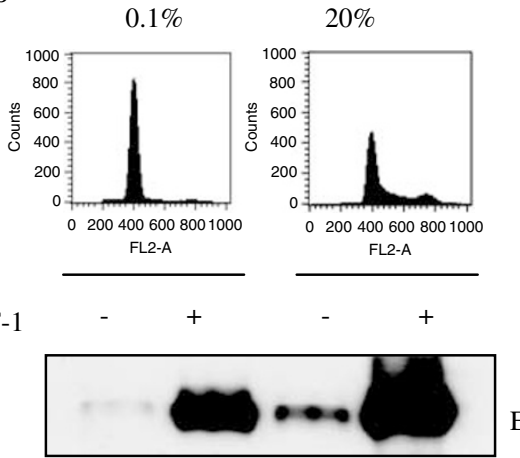

E2F-1

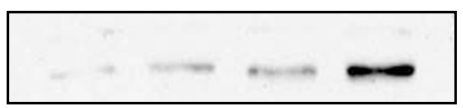

ASPP1

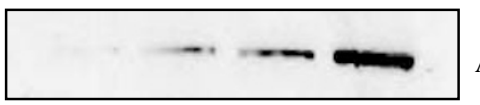

ASPP2

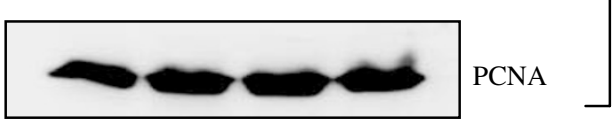

$\mathrm{wb}$

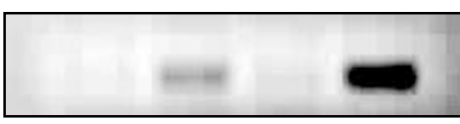

ASPP1

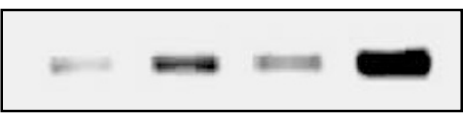

ASPP2

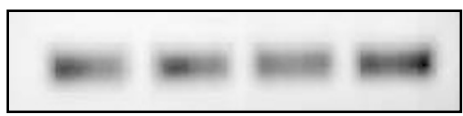

GAPDH

2

3

4

b

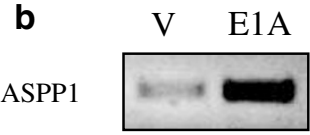

ASPP2

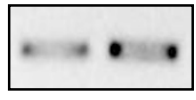

p73

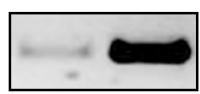

Actin twice with RIPA buffer, four times with IP washing buffer, ${ }^{30}$ twice with RIPA buffer and twice with $1 \times$ TE. In all, $20 \%$ of the supernatant from the rabbit IgG immunoprecipitation was saved as total input of chromatin, and was processed with the eluted immunoprecipitates beginning at the crosslink reversal step. Immunocomplexes were eluted for $10 \mathrm{~min}$ at $85^{\circ} \mathrm{C}$ in $125 \mu \mathrm{l}$ of $1 \% \mathrm{SDS} / 100 \mathrm{mM} \mathrm{NaHCO}_{3}$. A total of $0.1 \mu \mathrm{g} / \mu \mathrm{l}$ of salmon sperm DNA and $10 \mu \mathrm{g}$ of RNAse A were add to the pooled eluates and crosslinks were reverted by incubation for $6 \mathrm{~h}$ at $65^{\circ} \mathrm{C}$. Samples were diluted with $125 \mu \mathrm{l}$ of water containing $0.16 \mu \mathrm{g} / \mu \mathrm{l}$ proteinase $\mathrm{K}$, and incubated for $1 \mathrm{~h}$ at $50^{\circ} \mathrm{C}$. DNA was purified with phenol/chloroform and a fraction was used as PCR template to detect the presence of the promoter sequences of ASPP1 or ASPP2 ( 30 cycles of $30 \mathrm{~s}$ melting at $95^{\circ} \mathrm{C}, 30 \mathrm{~s}$ annealing at $60^{\circ} \mathrm{C}, 1 \mathrm{~min}$ extension at $68^{\circ} \mathrm{C}$ ) using the primers listed below:

\section{ASPP1: forward 5'-GGCTGGTTCCGAGCGCGCGGCCTT-3' reverse 5'-CCGCCTCAACCTCAGCCTCAGCCT-3' \\ ASPP2: forward 5'-TGGGGCGGCTCGGTGCCATTCACTA-3' reverse $5^{\prime}$-AGCGAGGCCGCCCGGACCTGTTGCG-3'}

PCRs were performed using the Taq PCRx polymerase kit from Invitrogen for GC-rich templates. PCR products were resolved in $1 \%$ agarose gels and visualised by ethidium bromide staining. The intensity of the PCR products was determined using the GeneTools software from Syngene.

\section{Transactivation assays}

Saos-2 cells $\left(8 \times 10^{5}\right)$ were seeded in $60 \mathrm{~mm}$ dishes and transfected the following day with $75-300 \mathrm{ng}$ of plasmid expressing E2F-1 together with $500 \mathrm{ng}$ of CMV- $\beta$-galactosidase ( $\beta$-gal) plasmid and $1 \mu \mathrm{g}$ of $3 \times$ wt-luc or luciferase reporter plasmids containing ASPP1 or ASPP2 promoter. At $24 \mathrm{~h}$ after transfection, cells were washed with PBS and lysed in RLT buffer (Promega) for Luciferase and $\beta$-gal assays.

\section{RT-PCR}

Total RNA was isolated from cells grown in culture dishes using the RNeasy ${ }^{\circledR}$ kit from Qiagen. cDNA was obtained using the SuperScript ${ }^{\mathrm{TM}}$ First-Strand Synthesis System (Invitrogen) following the manufacturer's instructions. PCR reactions of ASPP1, ASPP2 and GAPDH cDNA (25 cycles of $30 \mathrm{~s}$ melting at $95^{\circ} \mathrm{C}, 30 \mathrm{~s}$ annealing at $60^{\circ} \mathrm{C}, 30 \mathrm{~s}$ extension at $72^{\circ} \mathrm{C}$ ) were performed using the primers listed below:

\section{ASPP1 forward 5'-AGCAAGCCACACCACCTAAGAATTA-3' backward 3'-TGAACCCGAAGGTAAAACGGG-3' \\ ASPP2 forward 5'-GAAGACTCGGTGAGCATGCG-3' backward $5^{\prime}$-GCGATACGCTCTGAGCCAGT-3' \\ GAPDH forward 5'-CTCCTCCACCTTTGACGCTG-3' backward 5'-CCACCCTGTTGCTGTAGCCA-3'}

Figure 6 In vivo induction of ASPP1 and ASPP2 by elevated E2F activity. (a) Saos-2 cells expressing inducible E2F-1 were cultured for $48 \mathrm{~h}$ in DMEM containing 0.1 or $20 \%$ serum $(0.1 \%$ FCS or $20 \%$ FCS) as indicated. Their cell cycle status was analysed by FACS. Endogenous or overexpressed E2F-1 was detected by the anti-E2F-1 C-20 antibody (first panel). Total RNA extracted from Saos-2 cells grown in 0.1 or $20 \%$ FCS (a) or RPE cells expressing vector (v) or E1A (E1A) (b) was used for RT-PCR (last four panels) to detect the expression levels of ASPP1, ASPP2 and p73 mRNA. GAPDH expression was monitored in parallel as loading control. mASPP1/54.2 and mASPP2/54.10 antibodies were used to detect the expression of endogenous ASPP1 and ASPP2 proteins, respectively 


\section{Acknowledgements}

This work was funded by the Ludwig Institute for Cancer Research.

\section{References}

1. Hollstein M, Shomer B, Greenblatt M, Soussi T, Hovig E, Montesano R and Harris CC (1996) Somatic point mutations in the p53 gene of human tumors and cell lines: updated compilation. Nucleic Acids Res. 24: 141-146

2. Schmitt CA, Fridman JS, Yang M, Baranov E, Hoffman RM and Lowe SW (2002) Dissecting p53 tumor suppressor functions in vivo. Cancer Cell 1: 289-298

3. Ryan KM and Vousden KH (1998) Characterization of structural p53 mutants which show selective defects in apoptosis but not cell cycle arrest. Mol. Cell. Biol. 18: 3692-3698

4. Crook T, Marston NJ, Sara EA and Vousden KH (1994) Transcription activation by p53 correlates with suppression of growth but not transformation. Cell 79: 817-827

5. Ko LJ and Prives C (1996) P53: puzzle and paradigm. Genes Dev. 10: 1054-1072

6. Pan H, Yin C, Dyson NJ, Harlow E, Yamasaki L and Van Dyke T (1998) Key roles for E2F1 in signaling p53-dependent apoptosis and cell division within developing tumours. Mol. Cell 2: 283-292

7. Qin XQ, Livingston DM, Kaelin WGJ and Adams PD (1994) Deregulated transcription factor E2F-1 expression leads to S-phase entry and p53-mediated apoptosis. Proc. Natl. Acad. Sci. USA 91: 10918-10922

8. Wu X and Levine AJ (1994) p53 and E2F-1 cooperate to mediate apoptosis. Proc. Natl. Acad. Sci. USA 91: 3602-3606

9. Bates S, Phillips A, Clark P, Stott F, Peters G, Ludwig R and Vousden $\mathrm{KH}$ (1998) p14 ARF links the tumour suppressors RB and p53. Nature 395: 124-125

10. Weber JD, Taylor LJ, Roussel MF, Sherr CJ and Bar-Sagi D (1999) Nucleolar Arf sequesters Mdm2 and activates p53. Nat. Cell Biol. 1: 20-26

11. Tolbert D, Lu X, Yin C, Tantama M and Van Dyke T (2002) p19(ARF) is dispensable for oncogenic stress-induced p53-mediated apoptosis and tumor suppression in vivo. Mol. Cell. Biol. 22: 370-377

12. Tsai $K Y$, MacPherson D, Rubinson DA, Crowley $D$ and Jacks $T$ (2002) ARF is not required for apoptosis in Rb mutant mouse embryos. Curr. Biol. 12: 159-163

13. Samuels-Lev Y, O'Connor DJ, Bergamaschi D, Trigiante G, Hsieh JK, Zhong S, Campargue I, Naumovski L, Crook T and Lu X (2001) ASPP proteins specifically stimulate the apoptotic function of p53. Mol. Cell 8: 781-794

14. Bergamaschi D, Samuels Y, O'Neil NJ, Trigiante G, Crook T, Hsieh JK, O'Connor DJ, Zhong S, Campargue I, Tomlinson ML, Kuwabara PE and Lu X (2003) iASPP oncoprotein is a key inhibitor of p53 conserved from worm to human. Nat. Genet. 33: 162-167
15. Bergamaschi D, Samuels Y, Jin B, Duraisingham S, Crook T and Lu X (2004) ASPP1 and ASPP2: common activators of p53 family members. Mol. Cell. Biol. 24: $1341-1350$

16. Stanelle J, Stiewe T, Theseling CC, Peter M and Putzer BM (2002) Gene expression changes in response to E2F1 activation. Nucleic Acids Res. 30: 1859-1867

17. Kel AE, Kel-Margoulis OV, Farnham PJ, Bartley SM, Wingender E and Zhang $M Q$ (2001) Computer-assisted identification of cell cycle-related genes: new targets for E2F transcription factors. J. Mol. Biol. 309: 99-120

18. Phillips AC, Ernst MK, Bates S, Rice NR and Vousden KH (1999) E2F-1 potentiates cell death by blocking antiapoptotic signaling pathways. Mol. Cell 4 $771-781$

19. DeGregori J, Leone G, Miron A, Jakoi L and Nevins JR (1997) Distinct roles for E2F proteins in cell growth control and apoptosis. Proc. Natl. Acad. Sci. USA 94: $7245-7250$

20. Kowalik TF, DeGregori J, Leone G, Jakoi L and Nevins JR (1998) E2F1-specific induction of apoptosis and p53 accumulation, which is blocked by Mdm2 ${ }^{1}$. Cell Growth Differ. 9: 113-118

21. Lissy NA, Davis PK, Irwin M, Kaelin WG and Dowdy SF (2000) A common E2F1 and $p 73$ pathway mediates cell death induced by TCR activation. Nature 407: $642-645$

22. Vigo E, Muller $H$, Prosperini $E$, Hateboer $G$, Cartwright $P$, Moroni MC and Helin $\mathrm{K}$ (1999) CDC25A phosphatase is a target of E2F and is required for efficient E2F-induced S phase. Mol. Cell. Biol. 19: 6379-6395

23. Irwin M, Marin MC, Phillips AC, Seelan RS, Smith DI, Liu W, Flores IR, Tsai KY Jacks T, Vousden KH and Kaelin WG (2000) Role for the p53 homologue p73 in E2F-1-induced apoptosis. Nature 407: 645-648

24. Stiewe T and Putzer BM (2000) Role of the p53-homologue p73 in E2F1 induced apoptosis. Nat. Genet. 26: 464-469

25. Hsieh JK, Yap D, O'Connor DJ, Fogal V, Fallis L, Chan F, Zhong S and Lu X (2002) Novel function of the cyclin A binding site of E2F in regulating p53induced apoptosis in response to DNA damage. Mol. Cell. Biol. 22: 78-93

26. Moroni MC, Hickman ES, Denchi EL, Caprara G, Colli E, Cecconi F, Muller H and Helin K (2001) Apaf-1 is a transcriptional target for E2F and p53. Nat. Cell Biol. 3: 552-558

27. Furukawa $Y$, Nishimura N, Satoh M, Endo H, Iwase S, Yamada H, Matsuda M, Kano $Y$ and Nakamura M (2002) Apaf-1 is a mediator of E2F-1-induced apoptosis. J. Biol. Chem. 277: 39760-39768

28. Hershko T and Ginsberg D (2004) Up-regulation of Bcl-2 homology 3 (BH3)only proteins by E2F1 mediates apoptosis. J. Biol. Chem. 279: 8627-8634

29. Lowe SW, Ruley HR, Jacks T and Housman DE (1993) p53-dependent apoptosis modulates the cytotoxicity of anticancer agents. Cell 74: 957-967

30. Szak ST, Mays D and Pietenpol JA (2001) Kinetics of p53 binding to promoter sites in vivo. Mol. Cell. Biol. 21: 3375-3386

Supplementary Information accompanies the paper on Cell Death and Differentiation website (http://www.nature.com/cdd) 